\title{
Anatomy of the Opioid-Systems of the Brain
}

\author{
Karl M. Knigge and Shirley A. Joseph
}

This paper was presented in May 1983 at the Centennial Neurosciences Symposium of the Department of Anatomy, University' of Manitoba, at which Dr. Knigge was a keynote speaker.

In 1969, Roger Guillemin and Andrew Schally independently reported the isolation and identification of the first hypothalamic neuropeptide, thyrotropin releasing factor (TRF). Following this landmark event in neuroendocrinology the ensuing years have witnessed a cascade of isolations of new neuropeptides and a virtual revolution in neurobiology. The discipline of neuroendocrinology has been remarkably impacted by the evidence that all of the "hypophysiotrophic" releasing factors presently identified are distributed widely throughout the brain with neurotransmitter or neuromodulator roles quite different from their actions of regulating the secretion of pituitary hormones. The study of these neuropeptide systems in activity of the central nervous system looms as one of the most exciting and significant eras in brain research. Although it is premature to assign specific roles for the presently known neuropeptides in pathogenesis of neurological diseases, our limited current knowledge already points to a number of syndromes and clinical disorders which may be related to neuropeptide imbalance. Congential insensitivity to pain undoubtedly involves several peptide systems including Substance $P$, enkephalin, somatostatin and the endorphins. The opiocortins ( $\beta$-endorphin, ACTH) of the brain as well as those of the pituitary gland are directly involved in the homeostatic mechanisms brought into action by such trauma as brain and spinal cord injury, septic shock and hemorrhage. The role of peptides in regulation of cerebral circulation will likely be identified with the etiology of stroke and the production of painful hemicranial syndromes. Among the most prevelant disorders of the human nervous system are the dementias and psychoses (Alzheimer's disease, schizophrenia); subtle changes in brain peptide and receptor activity are being considered as responsible contributors to these diseases.

The opioid neuronal networks of the brain represent one of the most extensive and diverse neuropeptide systems thus far described and ones which have received perhaps the bulk of attention in clinical investigations. According to our present anatomical information, the central opioids are represented by at least 3 distinctly separate neuronal pools. These are (1) the opiocortin, (2) enkephalin, and (3) dynorphin groups. In our current classification, the opiocortin group is recognized as having 3 subpopulations: a hypothalamic arcuate opiocortin system, a brainstem medullary opiocortin pool of neurons, and a hypothalamic alpha MSH-specific system. In the present report we will review our anatomical studies on only the opiocortin division of the brain opioids. Unless specifically noted, our descriptions relate to the brain of the rat.

The arcuate opiocortin system consists of a pool or "bed nucleus" of perikarya located in the arcuate and periarcuate regions of the hypothalamus (Fig. 1). In species we have examined, including rat, mouse, hamster, guinea pig, dog, horse, primate and human, this pool of neuron cell bodies extends the entire antero-posterior distance of the hypothalamus. Medially, cell bodies are located along the lower $1 \mathrm{~mm}$ of the periventricular stratum. At all antero-posterior levels they are fairly uniformly distributed throughout the arcuate nucleus and extend laterally along the ventral floor as far as the region of the medial forebrain bundle. At the most anterior extent of the bed nucleus in the retrochiasmatic area and posteriorly in the mammillary region, the pool of perikarya bridges the midline (Knigge, Joseph and Nocton, 1981). Cell bodies are consistently present in the median eminence, located generally in the subependymal and internal zones (Knigge and Joseph, 1981). A quite remarkable fiber distribution originates from this pool of neurons (Joseph, 1980). The projections include intrahypothalamic formations, fibers to limbic structures, and a complex organization to the brainstem and spinal cord (Fig. 2). Figs. 3-6 illustrate the intrahypothalamic fiber distribution of this system at several levels in the rat brain. At the plane of the anterior commissure (Fig. 3), a particularily intense fiber innervation is present in the medial preopticperiventricular region. The association of this area with desending autonomic pathways involved in control of blood pressure may provide, in part, the anatomical substrate for central opiocortin involvement in a variety of parameters of cardiovascular function. At the level of the anterior commissure also, the nucleus stria terminalis, particularily its ventral subdivisions, receives a dense fiber innervation. At the level of the optic chiasm (Fig. 4) the anterior-most portion of the paraventricular nucleus has a notable opiocortin fiber density, while the suprachiasmatic and supraoptic nuclei are conspicuously fiber-free. At subsequent levels of the 


\section{List of Abbreviations}

\author{
aaa — anterior amygdaloid area \\ ac - anterior commissure \\ abl - amygdala, basolateral nucleus \\ aco - amygdala, cortical nucleus \\ ah - anterior hypothalamic area \\ al - amygdala. lateral nucleus \\ am - amygdala. medial nucleus \\ aq - cerebral aqueduct \\ arc - arcuate nucleus \\ c - central canal \\ ca - anterior commissure \\ cc - corpus callosum \\ cfv - fornix, commissure \\ ci - internal capsule \\ cl - claustrum \\ co - optic chiasm \\ cp - cerebral peduncle \\ dh - dorsal hypothalamus \\ dif - dorsal longitudinal fasciculus \\ dm - dorsomedial nucleus \\ dmd- dorsomedial nucleus, pars dorsalis \\ $\mathrm{dmv}$ - dorsomedial nucleus, pars ventralis \\ dr — dorsal raphe nucleus \\ ew - Edinger-Wesphal nucleus \\ fc - fasciculus cuneatus \\ fldg - dorsal longitudinal fasciculus, pars tegemtalis \\ fldk - dorsal longitudinal fasciculus, pars tectalis \\ flm - medial longitudinal fasciculus \\ fr - fasciculus retroflexus \\ fx - fornix
}

gd - dentate gyrus

hi - hippocampus

hp - posterior hypothalamus

inf - infundibulum

ip - interpeduncular nucleus

in - lateral habenular nucleus

tha - lateral hypothalamic are

Im - medial lemniscus

Ipo - lateral preoptic area

It - lateral tegmental nucleus

me - median eminence

mg - medial geniculate body

$\mathrm{mh}$ - medial habenular nucleus

mlf - medical longitudinal fasciculus

$\mathrm{mm}$ - mammillary nuclei

mpo-medial preoptic area

mr - medial raphe nucleus

$\mathrm{mt}$ - mammillothalamic tract

not - nucleus, olfactory tract

ncom

- nucleus tractus solitarius, pars commissuralis

$\mathrm{nmt}$ - nucleus mesencephalic tract of $\mathrm{V}$

npd - nucleus parabrachialis, pars dorsalis

nph - nucleus prepositus hypoglossi

npv - nucleus parabrachialis, pars ventralis

nst - nucleus stria terminalis

ntd - dorsal tegmental nucleus

nvm- medial vestibular nucleus

ot - optic tract

pag - periaqueductal gray pc - posterior commissure

pcs - superior cerebellar peduncle

pd - pituitary gland. pars distalis

pi - pituitary gland, pars intermedia

pir - piriform cortex

pl - pituitary gland. pars nervosa

po - preoptic area

pt - pretectal nucleus

pvn - paraventricular nucleus

pvt - thalamus, periventricular nucleus

II - nucleus lineris rostralis

sc - superior colliculus

si - inferior colliculus

- lateral septal nucleus

so - supraoptic nucleus

sol - nucleus tractus solitarius

sm - stria medullaris

smn - medial septal nucleus

snr - substantia nigra

t - stria terminalis

tad - thalamus, nucleus anterior dorsalis

th - thalamus

is - iractus solitarius

$v_{3}-3$ rd cerebral ventricle

vl - lateral cerebral ventricle

$\mathrm{Vmb}-$ ventromedial nucleus

$\mathrm{Vr}$ - recess. 3rd cerebral ventricle

XII - hypoglossal nucleus

zi - zona incerta

th

Figure I - Frontal section of rat brain. The arcuate opiocortin system is immunostained with anti-ACTH. Cell bodies in the arcuate mucleus (arc) are prominent because of colchicine pre-treatment. The thalamus (th). except for fibers of the fasciculus (dlb) in mucleus periventricularis, is relatively free of opiocortin innervation. X20. 


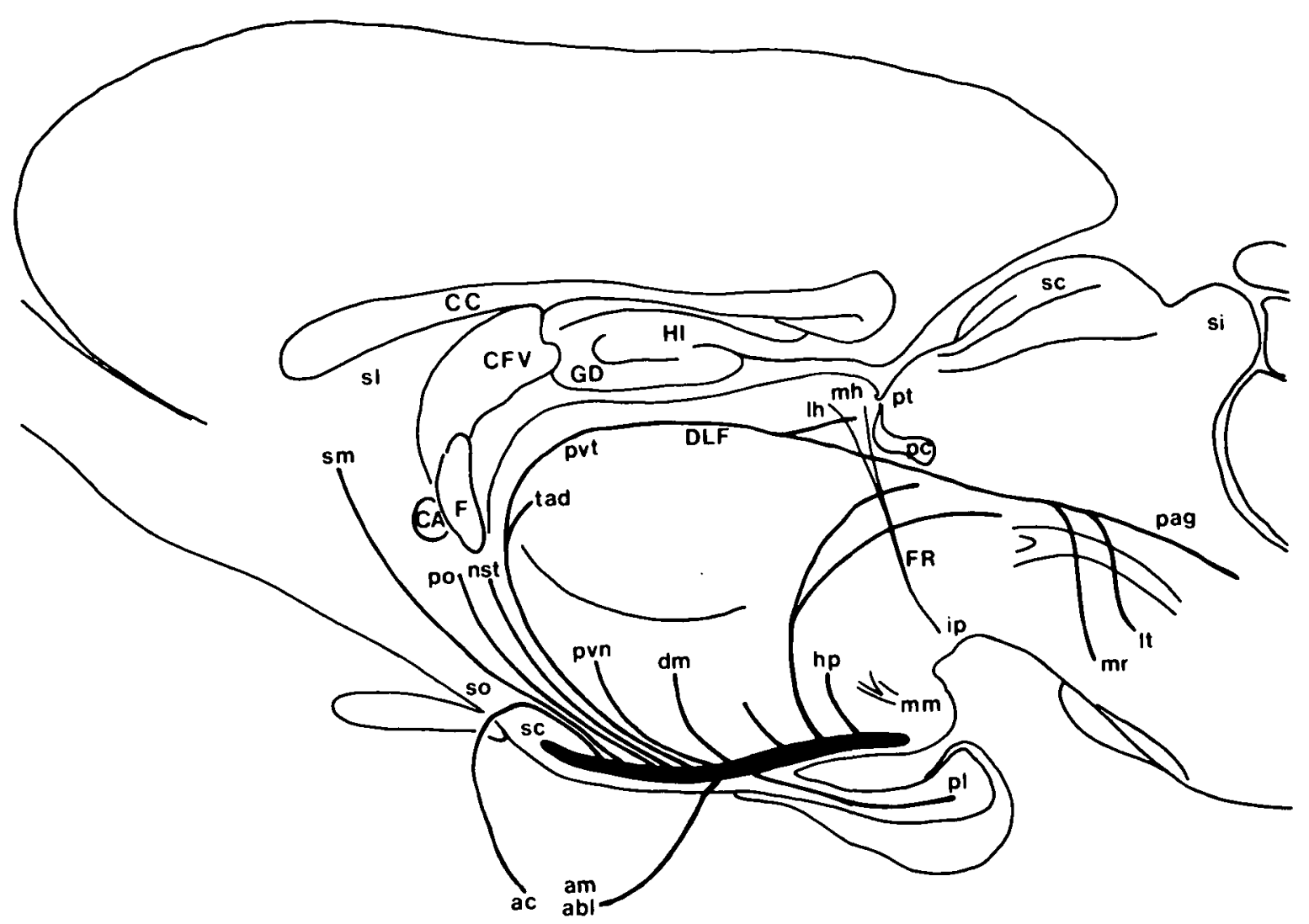

Figure 2 - Midsaggital representation of the rat brain illustrating the major efferent fiber projections from the arcuate opiocortin "bed nucleus", shown as the heavy solid area in the ventral hypothalamus. The primary domains of these neurons include intrahypothalamic nuclei, the posterior lobe of the pituitary gland. several amygdaloid nuclei, anterior and medial nuclei of the thalamus, and extensive centers in the brainstem.

paraventricular nucleus (Fig. 5), both its parvo-and magnocellular portions are heavily innervated. Details of the relationships of opiocortin fibers to oxytocin- and vasopressin-containing neurons here and in the supraoptic nucleus have been described by Knigge and Joseph (1982) and Sawchenko, Swanson, and Joseph (1982). In mid-hypothalamus (Fig. 6) the contrast in innervation between the dorsomedial and ventromedial nuclei is striking. In our experience, both dorsal and ventral divisions of the dorsomedial nucleus are the most heavily innervated hypothalamic region. This striking opiocortin input may hopefully stimulate renewed interest in this most "silent" nucleus of the hypothalamus. Projections to the neurohypophysis of the rat proceed into the internal zone of the median eminence, descend into the stalk and appear to distribute fairly uniformly throughout the neural lobe (Knigge and Joseph, 1981). This projection is most conspicuous in the dog brain (Figs. 7, 8). An influence of opiocortin peptides in neural lobe (oxytocin and vasopressin) functions as well as the presence of opiate receptors in the posterior pituitary have been amply documented; the anatomical groundwork for a mechanism of action of opiocortin peptides in the neural lobe clearly exists, but awaits further studies at the fine structural level to reveal the synaptic relationships of these projections with the oxytocinergic and vasopressinergic fibers which terminate here. The heavy opiocortin innervation of the paraventricular nucleus and the virtual absence of this system in the supraoptic nucleus adds an interesting dimension to the issue of opiocortin influence on magnocellular function and would appear to leave the neural lobe as a likely site of action.
Several nuclei of the amygdala represent major termini of the arcuate opiocortin system. Fibers from perikarya of the bed nucleus are routed in two distinct pathways to reach these limbic areas (Fig. 9). Anterior projections along the periventricular stratum turn sharply at the approximate level of the anterior commissure, sweep laterally and posteriorly through the preoptic area and substantia inominata; these fibers appear to distribute predominantely to the central nucleus of the amygdala. A second projection courses in part in the classical ventral amygdalofugal pathway; from arcuate cell bodies, fibers course through the lateral hypothalamus, arch over the ascending optic tract and reach the central, basolateral and dorsal portions of the basomedial amygdaloid nuclei. No arcuate opiocortin fibers have been detected in the stria terminalis nor in any portion of the hippocampal complex. Relatively little information is available regarding the functional role of these amygdaloid opiocortin projections.

Analysis of the opiocortin projections to brainstem and spinal cord reveals innervations within regions containing catecholamine systems, areas associated with regulation of sympathetic and parasympathetic outflow, cardiovascular and respiratory homeostatic centers and regions associated with pain modulation (Romagnano and Joseph, 1983). Most if not all areas receiving opiocortin fibers have been shown to contain opiate receptors, suggesting that there exists an anatomical substrate for functional integration of the endogenous opioid system.

It is uncertain at this time whether the cells responsible for all of the brainstem projections reside within the hypothalamic 
Figures 3-6 - Frontal sections of rat brain, immunostained with anti-ACTH, at representative levels of the hypothalamus to illustrate details of the opiocortin fiber distribution. X30.

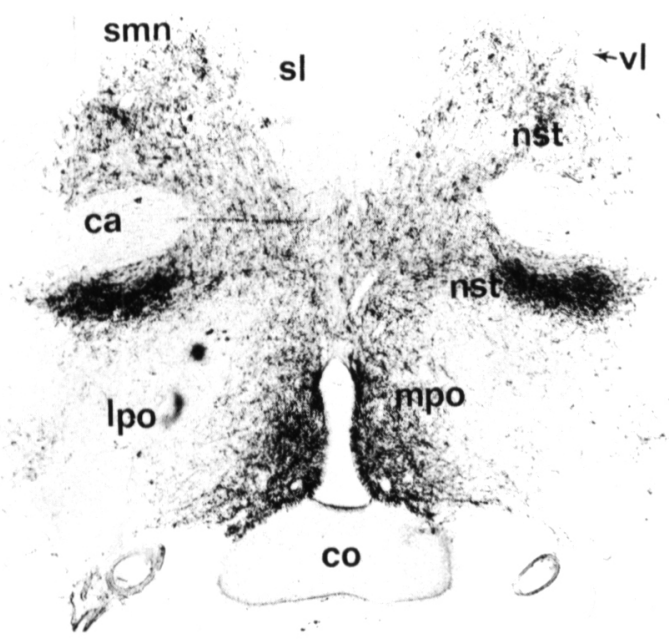

Figure 3 - At the level of the anterior commissure (ca), the medial preoptic area (mpo) and ventral divisions of nucleus stria terminalis (nst) are heavily innervated.

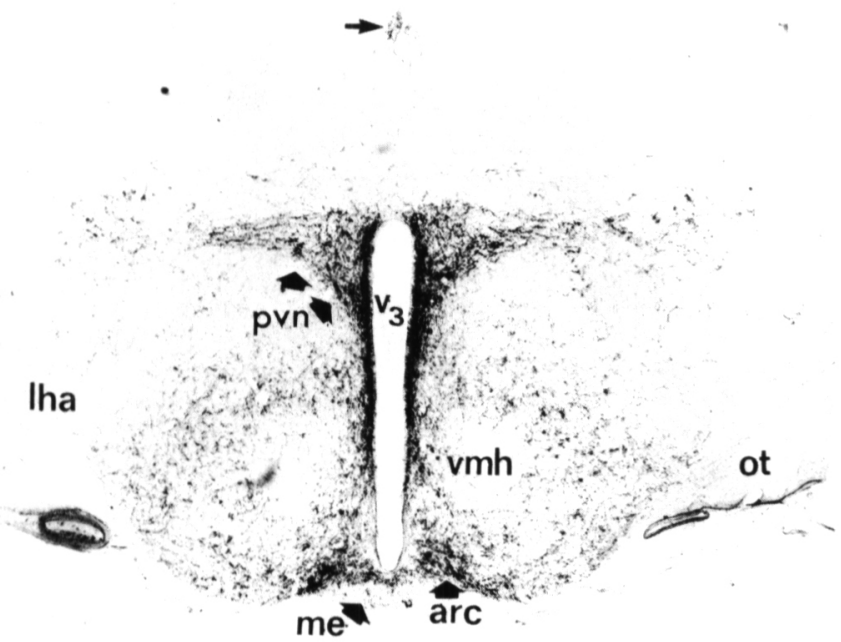

Figure 5-Innervation of the paraventricular nucleus (pvn) and adjacent periventricular stratum is apparent. In non-colchicine treated animals, opiocortin perikarya are embedded in dense networks of fibers in the arcuate nucleus (arc) and are less readily visible. A small bundle of fibers $\rightarrow$ represents contributions to the posterior division of the dorsal longitudinal fasciculus.

arcuate bed nucleus (as described above) or emanate from a group of perikarya localized in the medulla which we have recently designated as the medullary opiocortin nucleus (Joseph, Pilcher and Bennett-Clarke, 1983). Based on previous (Scott and Knigge, 1981) and current hypothalamic deafferentation studies (Michael and Joseph, 1983) it appears that the fiber distribution from the hypothalamic arcuate neurons extends to caudal pontine levels, while the remaining brainstem and spinal cord are innervated by the medullary opiocortin nucleus.

One of the main pathways by which the opiocortin fibers reach caudal diencephalon and brainstem is via the dorsal longitudinal fasciculus of Schütz (DLF) [Joseph, 1980; Fig. 1].

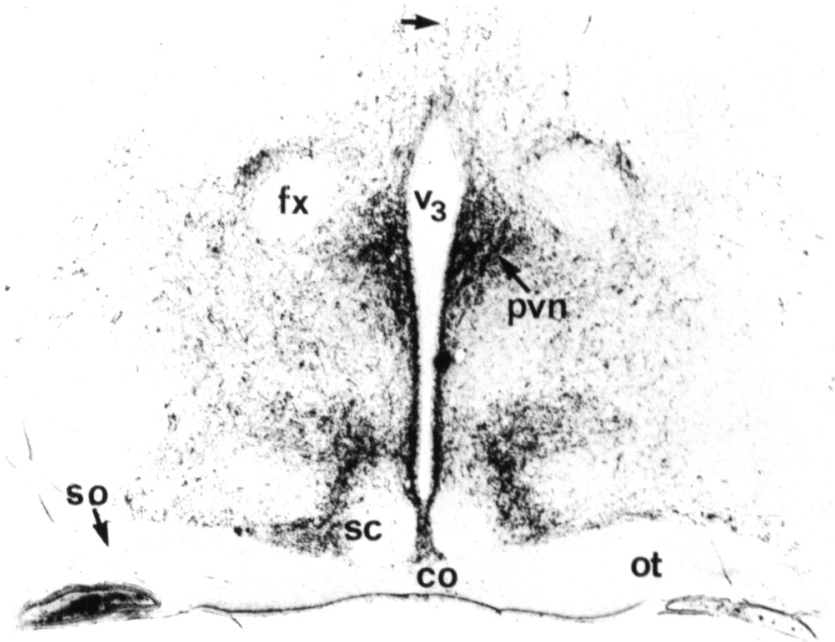

Figure 4-At the level of the optic chiasm (co) the absence of opiocortin fibers in the supraoptic (so) and suprachiasmatic (sc) nucle is conspicuous. Fibers ascending in the thalamic midline $\rightarrow$ are contriburing to the formation of the anterior division of the dorsal longitudinal fasciculus.

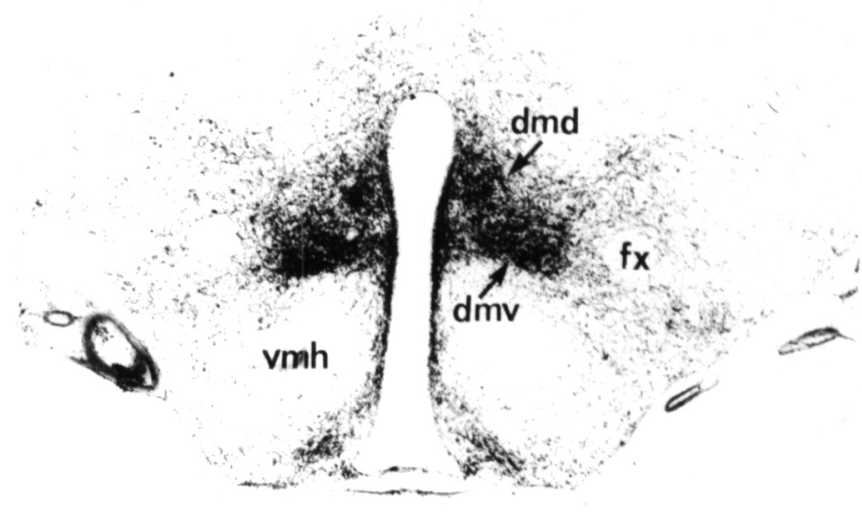

Figure 6 - The intense fiber concentration in both divisions of the dorsomedial nucleus (dmd, dmv) is in stark contrast to the paucity of fibers in the ventromedial nucleus (vmh).

This bundle consists of an anterior (periventricular) and a posterior division. The latter divides into tectal and tegmental components in the mesencephalic periaqueductal gray (PAG). The arcuate opiocortin fibers of the DLF course anteriorly in the periventricular stratum and ascend in diffuse fascicles in the anterior hypothalamic and preoptic regions. In the vicinity of the precommissural fornix columns these fibers condense dramatically into bilateral, dense bundles which arch upward and innervate nucleus periventricularis thalami, nucleus anterior dorsalis thalami and at the level of the habenula, fibers are present in the margin between the medial and lateral habenular nuclei. The major projection site for opiocortin fibers in the posterior division of the DLF is the brainstem. 
In the mesencephalon at the level of the posterior commissure and medial geniculate body opiocortin fibers are found in the pretectal area and in discrete bundles in the periaqueductal gray, encircling the medial geniculate body, in the lateral tegmental nucleus, the ventral tegmental nucleus of Tsai and in the midbrain tegmentum dorsal to the substantia nigra. The distribution of opiocortin fibers within the periaqueductal gray is of interest in view of their anatomical organization as well as their implied functional significance. At the most rostral level of the cerebral aqueduct fibers in the PAG are parceled into a dorsal median group, a ventral median group and distinct ventro-lateral bundles (Fig. 10). The ventro-lateral bundle represents the tegmental division of the posterior component of the DLF. At the level of the superior colliculus fibers of the DLF extend into the lateral tegmental area and mesencephalic reticular formation where fibers merge with the perigeniculate fibers. Further caudally at the rostral extent of the inferior colliculus this lateral tract separates into discrete upper and lower divisions which project even further into the reticular formation and ventral tegmental area where they eventually merge (Fig. 11). This trajectory appears to be coincident with ascending catecholamine pathways. As the aqueduct widens at mid to caudal inferior collicular levels, PAG fibers remain densely packed surrounding the aqueduct. At the level of the fourth ventricle, opiocortin fibers are particularily dense in the locus ceruleus and parabrachial nuclei.

One of the most intensely studied and to date best understood functions of the central endogenous opioids are their participation in the modulation of endogenous pain control systems. Myriad neuroanatomical, electrical physiologic and intracerebral injection studies have amply demonstrated that the periaqueductal gray region is a focal point for the integration of the effects of endogenous opioids. The pioneering studies of Reynolds in 1969 demonstrated that stimulation of periaqueductal gray produced sufficient analgesia in rats to allow surgery to be performed without the use of anesthetics. It has been shown that the ventro-lateral PAG is especially sensitive to opiate and opioid application and that lesions in this area result in a hyperalgesic state. It is significant therefore that we have demonstrated within this opiate-opioid sensitive area, rich in opiate receptors, very discrete and heavy innervations of opiocortin fibers.

The brainstem opiocortin fibers exhibit a very obvious co-localization with numerous noradrenergic and serotonergic cell groups. These include: nucleus cuniformis and the lateral tegmental area $\left(A_{8}\right)$, nucleus linearis pars intermedius $\left(A_{10}\right)$, the area ventral to brachium conjunctivum and the ventral parabrachial nucleus $\left(A_{7}\right)$, locus ceruleus $\left(A_{6}\right)$, lateral reticular nucleus adjacent to the emerging facial nerve and medial to the motor division of $V\left(A_{s}\right)$, the area ventral and lateral surrounding the 4th ventricle $\left(A_{4}\right)$, dorsal to the dorsal accessory olivary nucleus $\left(A_{3}\right)$, in nucleus and tractus solitarius $\left(A_{2}\right)$, and in the lateral reticular nucleus $\left(A_{1}\right)$. Opiocortin fibers are also present in serotonin groups: nucleus raphe dorsalis (B6-B7), nucleus centralis superior (B8-B9), nucleus raphe pontis (B5), nucleus raphe magnus (B3), and nucleus raphe obscurus (B2). It has been demonstrated that monoaminergic systems are involved in analgesic mechanisms; although our observations at the light microscope level do not demonstrate a functional interaction, it is tempting to speculate that there exists an interplay between the opiocortin and certain monoaminergic systems.

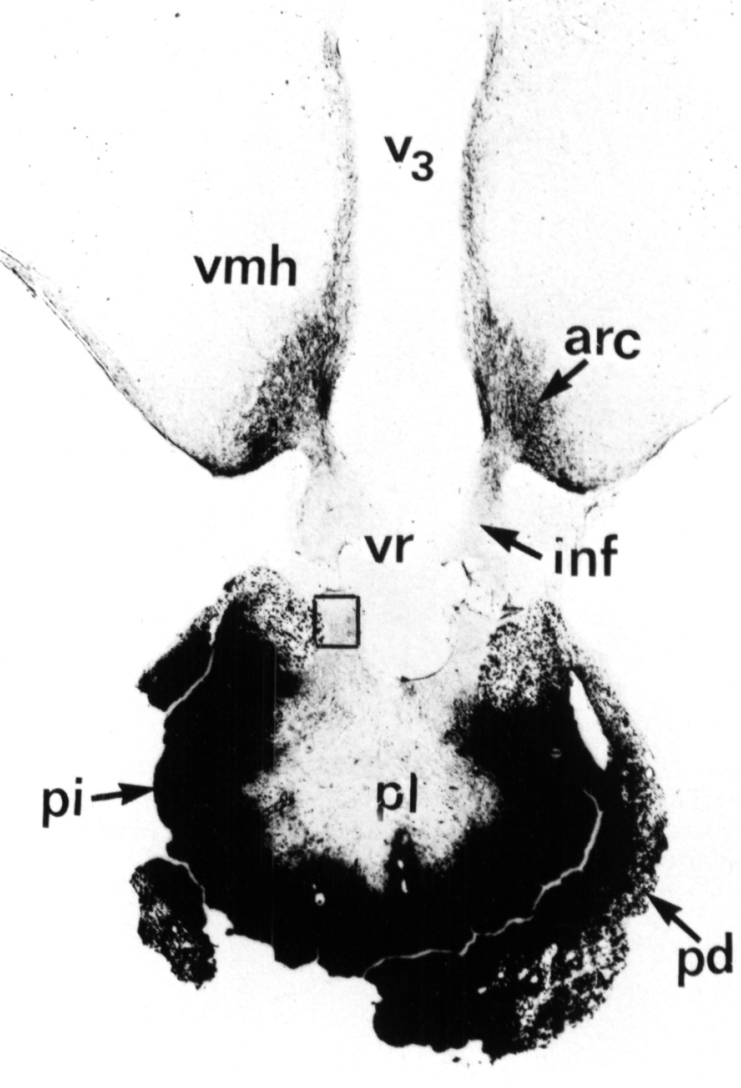

Figure 7 - Frontal section of dog hypothalamus with pituitary glandattached. Opiocortin perikarya are wedged between extremely delicate and tightly packed fibers in the arcuate periarcuate region (arc). From here, fibers descend into the infundibulum (inf) and distribute in the posterior lobe (pl). Details of this, in the area outlined, are shown in Fig. 8. At the concentrations of ACTH antiserum used to reveal the brain opiocortin system, cells of the pars intermedia (pi) and corticotrophs of the anterior lobe $(p d)$ are prodigiously overstained. $X / 3$

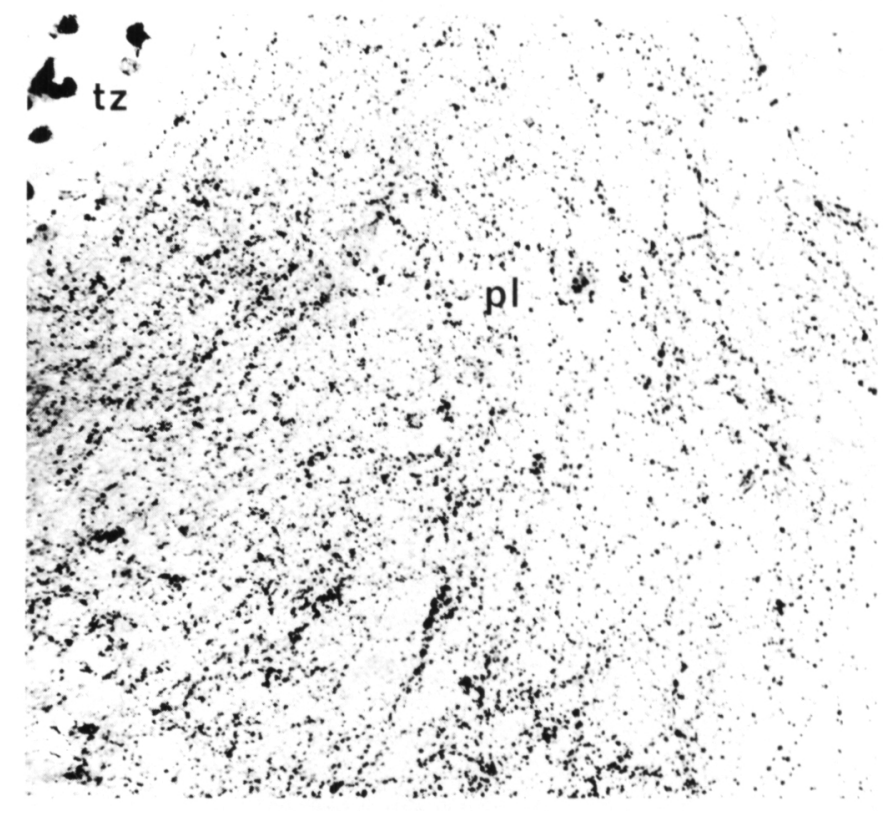

Figure 8 -Details of the opiocortin distribution in the posterior lobe (pl) of the dog pituitary gland. A very dense plexus of fibers and terminals is distributed fairly uniformly throughout the lobe. $t z=$ transition zone between the anterior lobe and pars intermedia. X200. 
Medullary Opiocortin System. As noted previously, we have identified a new group of opiocortin neurons in the medulla, within the commissural division of nucleus tractus solitarius. By virtue of their location within this cardiovascular and respiratory homeostatic center, these neurons may provide an additional, perhaps more select circuit of opiocortin influence in visceral/autonomic responses.

The greatest concentration of these perikarya are within the nucleus commissuralis tractus solitarius, medial and ventral to tractus solitarius and scattered along a ventro-lateral axis through nucleus reticularis medullae to the border of nucleus ambiguus (Fig. 12). These neurons are bipolar and measure $10-30 \mu \mathrm{m}$ in diameter. Their rostral-caudal extent is from the caudal boundry of the area postrema to the first and second cervical cord levels; here they surround the central canal. To date we have seen these cells only in the colchicine treated rat. Although the precise extent of the fiber projections from these cells is not as yet known it is apparent that the lower medulla and spinal cord receive their innervation from the medullary opiocortin neurons. In the medulla processes are observed within all divisions of nucleus tractus solitarius throughout its rostral-caudal extent, within and surrounding the area postrema. At more caudal medullary levels fibers project in a ventral-lateral direction through the nucleus reticularis medullae oblongata, nucleus ambiguus, and nucleus reticularis lateralis $\left(A_{1}\right)$. Some fibers are also present in nucleus reticularis gigantocellularis and at all levels of the spinal cord surrounding the central canal.
The Opiocortin System and CRF. Of the several interactions which the opiocortin systems have with other neuropeptides, that with the corticotropin releasing factor (CRF) is provocative in its functional implications. Four decades of effort to identify this important peptides culminated recently in the isolation by Wyle Vale and his colleagues of a 41 amino acid polypeptide from ovine hypothalami. We (Joseph and Knigge, 1983) and others have developed antibodies to CRF ${ }^{1-41}$ and have described the distribution of perikarya and fibers throughout brain and spinal cord. In further studies, we (Pilcher and Joseph, 1983) have recognized a unique co-distribution of CRF perikarya and opiocortin fibers. The entire pool of CRF cell bodies in the brain appears to be heavily innervated by the arcuate and medullary opiocortin systems.

In the forebrain and rostral diencephalon these neuronal elements are co-localized in nucleus accumbens, surrounding the precommissural and commissural fibers of the anterior commissure, in the lateral septum, and in stria terminalis. Dense accumulation of CRF perikarya and opiocortin fibers are observed along the periventricular stratum from the suprachiasmatic nucleus to the level of the fornix where they spray laterally to encompass this tract. In the lateral hypothalamus and preoptic regions this co-distribution is also apparent as scattered CRF perikarya are intermixed with opiocortin fibers. As previously described a very dense innervation of opiocortin fibers is present in the paraventricular nucleus; this nuclear complex contains perhaps the most dense aggregation of $C R F$ containing neurons

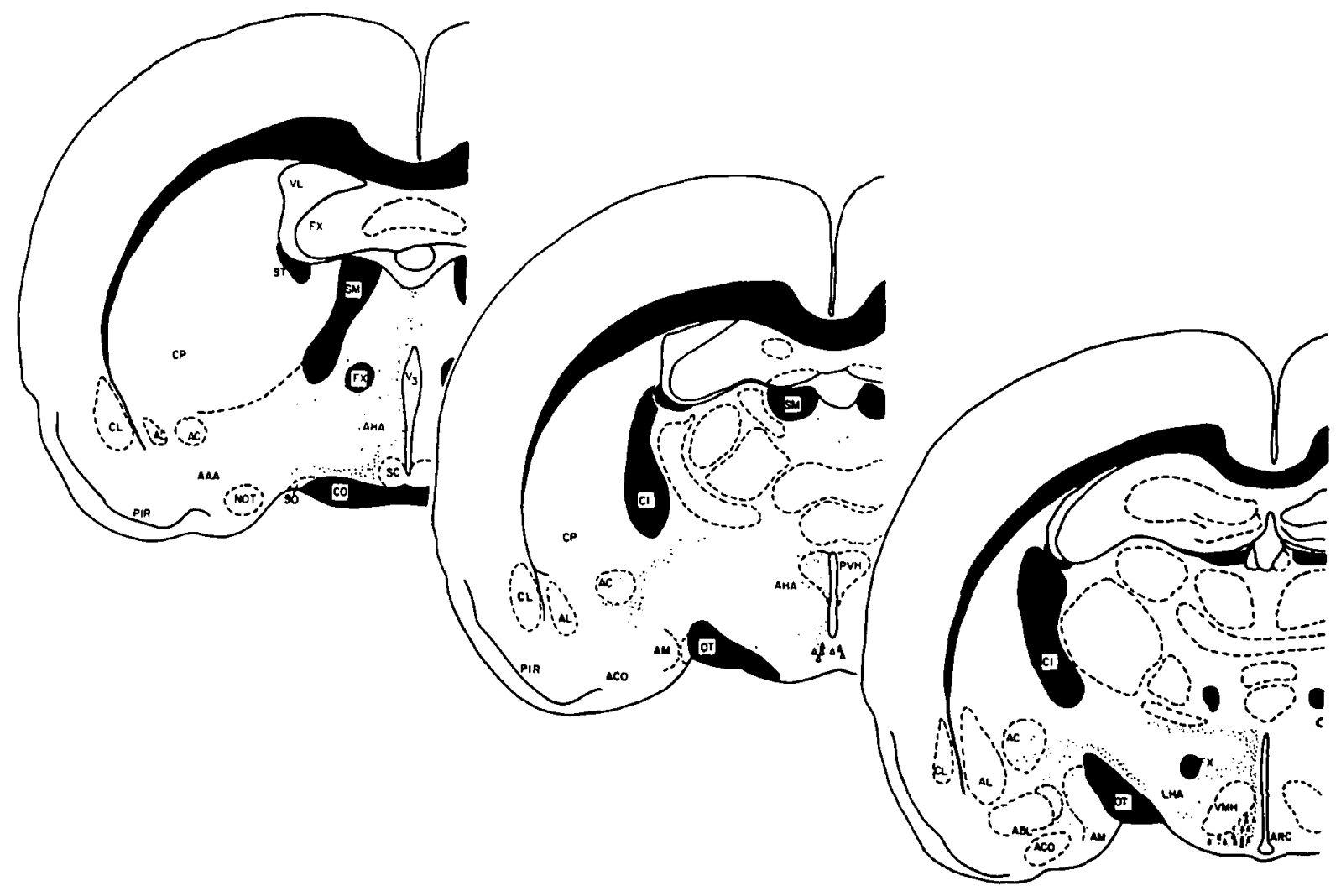

Figure 9 - Schematic frontal sections of rat brain illustrating the 2 pathways of arcuate opiocortin projections to the amygdala. The anterior traverse involves fibers which sweep through the anterior hypothalamic (aha), preoptic and amygdaloid (aaa) areas, innervating primarily the central amygdaloid nucleus. Fibers in the lateral bundle proceed in the ventral amygdalofugal pathw'a. cross the optic tract and distribute to medial. basolateral and the central muclei. 
(Fig. 13). In addition, the central and to a lesser extent the medial nuclei of the amygdala contain CRF perikarya and fibers.

In the posterior hypothalamus the co-distribution of CRF perikarya and opiocortin fibers is apparent throughout the mammillary complex. In thalamus these elements are present in nucleus reuiens, in the perifornical region, in nucleus ventralis medialis thalami and in nucleus subparafascicularis.

In the mesencephalon CRF cells are observed in conjunction with opiocortin fibers surrounding the medial geniculate body and in the lateral tegmental nucleus ( $A_{8}$ catecholamine area), along the dorsal border of substantia nigra and within the reticular formation (Fig., 14a). The density of CRF neurons in PAG is minimal and observed mainly in conjunction with the opiocortin fibers in the ventral-lateral division of the DLF. Also at this level CRF perikarya and opiocortin fibers are observed in the nucleus raphe dorsalis, nucleus lineralis rostralis, and in the region of the Edinger-Westphal nucleus. A pronounced codistribution pattern of CRF perikarya and opiocortin fiber is seen in caudal medulla: ventral to the ventral tip of the brachium conjunctivum $\left(A_{7}\right)$, in the Kolliker Füse respiratory nucleus, parabrachial nucleus, locus ceruleus $\left(A_{6}\right)$, dorsal tegmental nucleus and nucleus raphe pontis $\left(B_{5}\right)(F i g .14 b, c)$.

In caudal medulla CRF cells associated with opiocortin fibers are prominent in the area of the nucleus prepositus hypoglossi and median vestibular nucleus, lateral to the medial leminiscus and inferior olive, in the nucleus ambiguus, and associated with fibers of the spinothalamic tract (Fig. 14d). Within the nucleus tractus solitarius these elements are also present and are most abundant in the commissural portion of the nucleus where the medullary opiocortin cells have been localized. From this localization opiocortin fibers and CRF perikarya extend ventrolaterally across the reticular formation into the lateral reticular nucleus $\left(A_{1}\right)$ (Fig. 14e). Co-localization of these elements in the first few cervical cord sections is confined to a few cells and fibers surrounding the central canal.

Neuropeptides of the Opiocortin System. The neurons of the opiocortin system described above synthesize a family of peptides, among which are the biologically active substances ACTH, $\alpha \mathrm{MSH}$, and $\beta$-endorphin. These neurons appear to share a common biosynthetic process with corticotrophs of the adenohypophysis and the cells of the pars intermedia. All three synthesize a large 31 kilodalton precursor molecule (Fig. 15). The $31 \mathrm{~K}$ precursor molecule of the adenohypohysis has been derived from the sequence of cloned cDNA and its amino acid sequence analyzed. Post-translation processing of the $31 \mathrm{~K}$ precursor involves glycosylation and several proteolytic cleavages resulting in 2 major intermediate products, $23 \mathrm{~K}$ intermediate and $\beta$-lipotropin. In the adenohypophysis, subsequent cleavages yield ACTH and $\beta$-endorphin as active secretory products which are both released into the systemic circulation. Whether all corticotrophs possess and obligatory path for release of equimolar amounts of these 2 peptides under normal or stimulated conditions is not clearly defined. In the pars intermedia, the 1-13 N-terminal sequence of ACTH is cleaved to yield this tissue's major active hormone, $\alpha-\mathrm{MSH}$; the residue of this cleavage is CLIP (corticotropin-like intermediate peptide). The disposition and biological role of the additional peptide fragments of this opiocortin biosynthetic sequence are at present not well established.

The events of this process in the opiocortin neurons are less well understood than in pituitary gland. Based upon radioim-

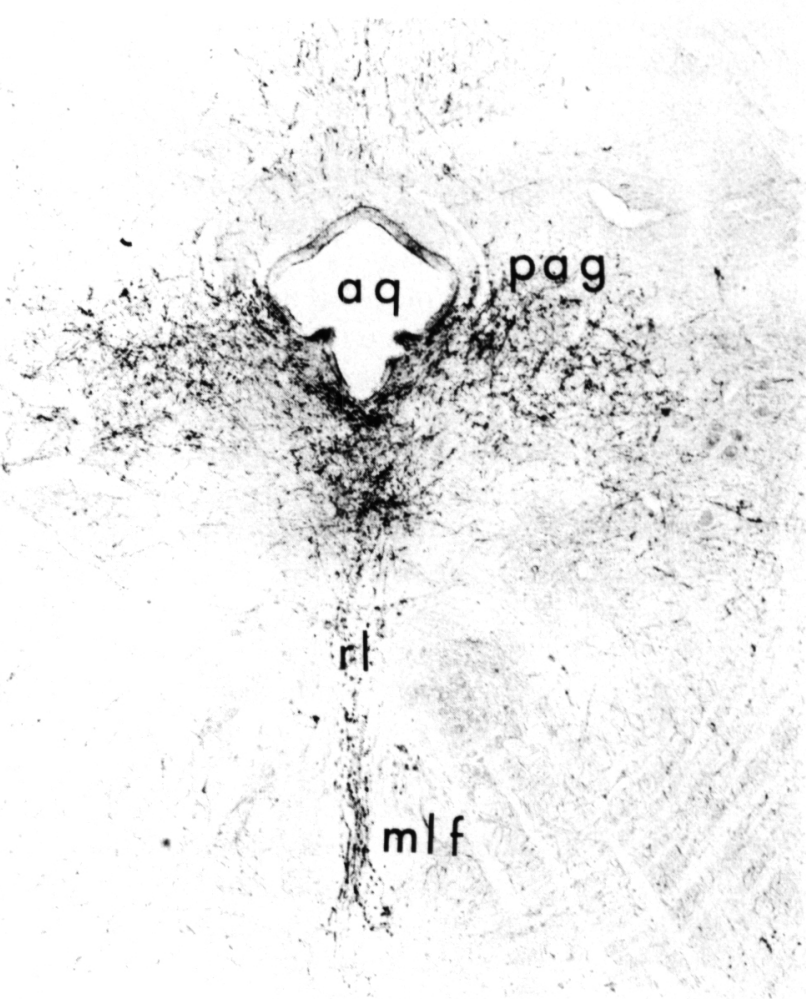

Figure $10-$ Distribution of opiocortin fibers in the periaqueductal gray (pag) of the rostral mesencepahlon. X35.

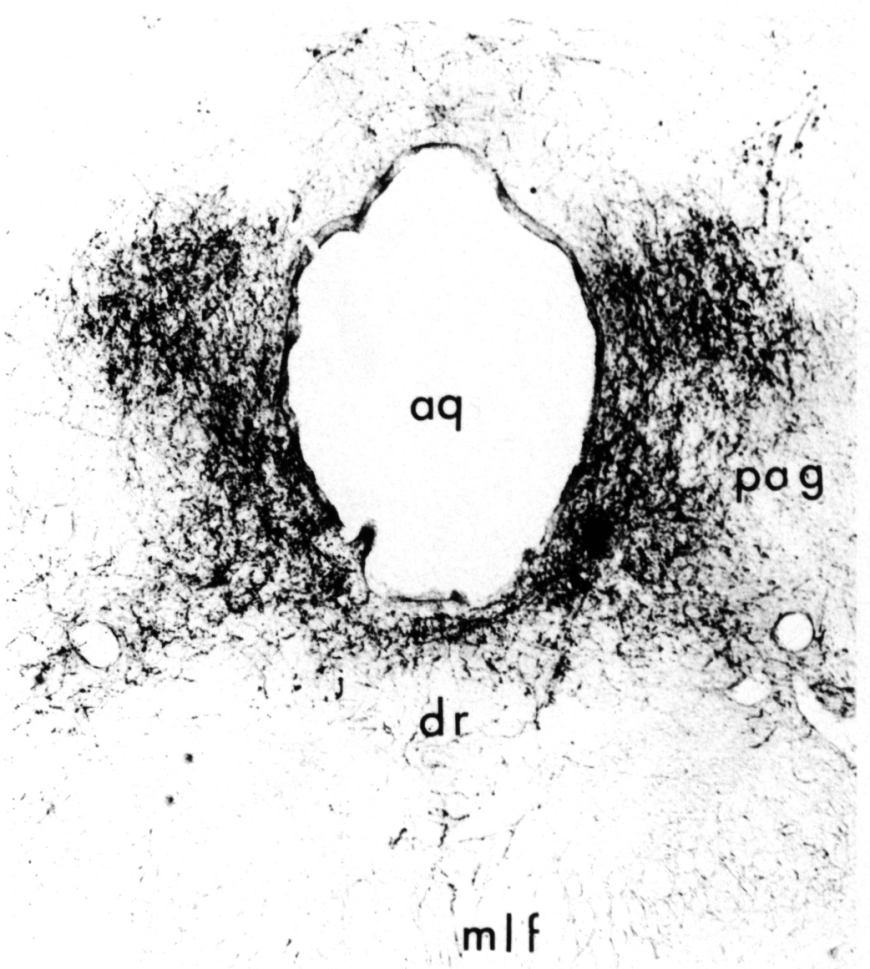

Figure $11-$ At the level of the inferior colliculus, lateral bundles of opiocortin fibers in the periaqueductal gray (pag) separate into distinct upper and lower divisions; both project extensively into lateral and ventral tegmental areas. X35. 


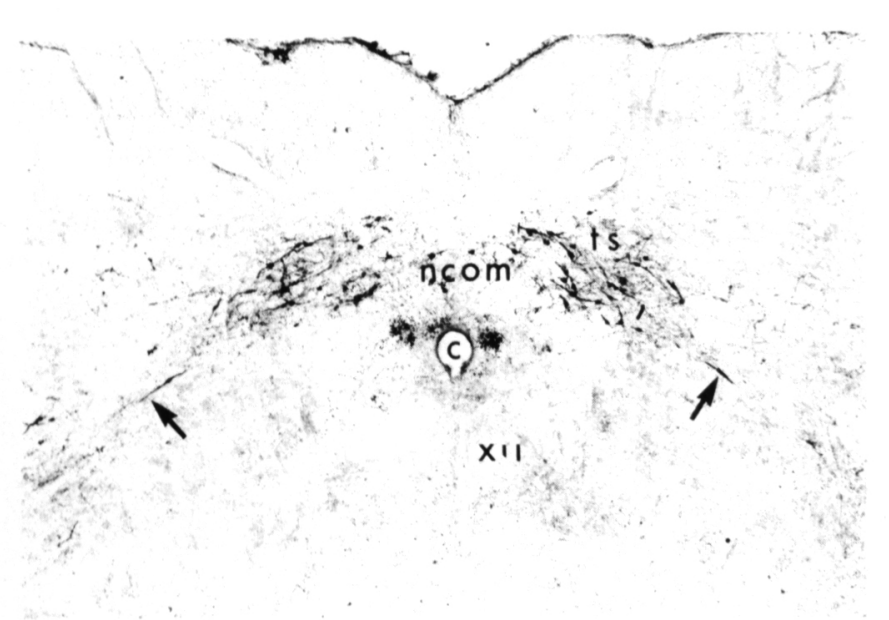

Figure $12-$ Neurons of the medullary opiocortin nucleus. Cell bodies are located in the commissural portion of nucleus tractus solitarius (sol) and extend ventrolaterally $\rightarrow$ into the Al catecholamine region. $X 35$.

a
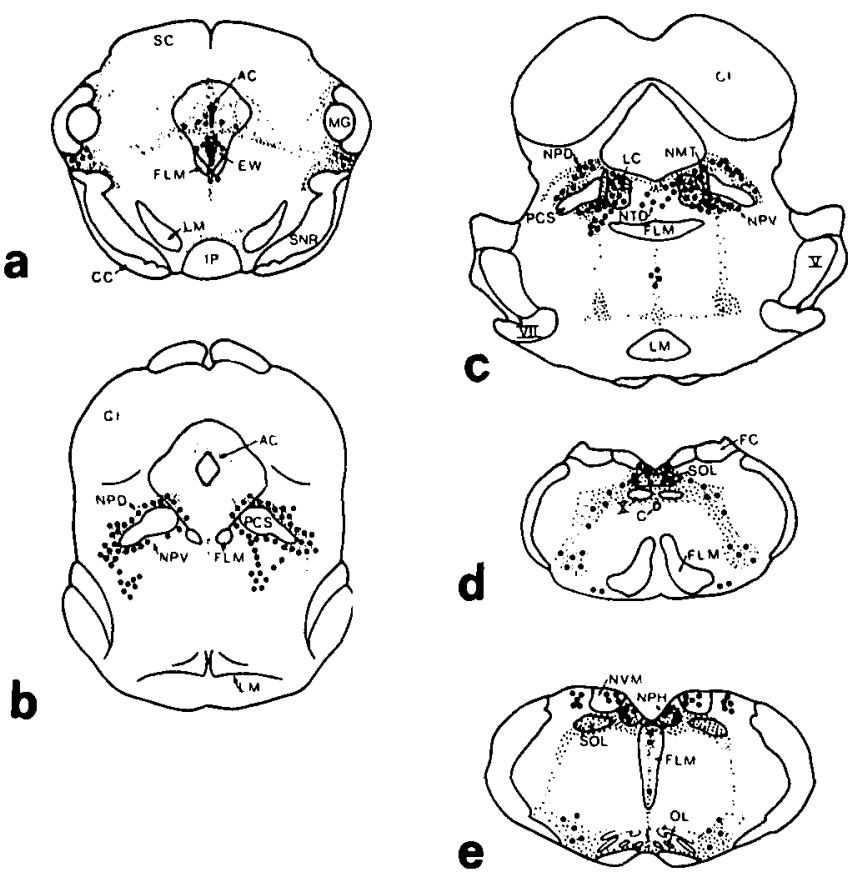

Figure 14 - Schematic figuresillustrating the co-localization of CRF perikarva (heavy dots) and opiocortin fibers (stipple) in the brainstem.

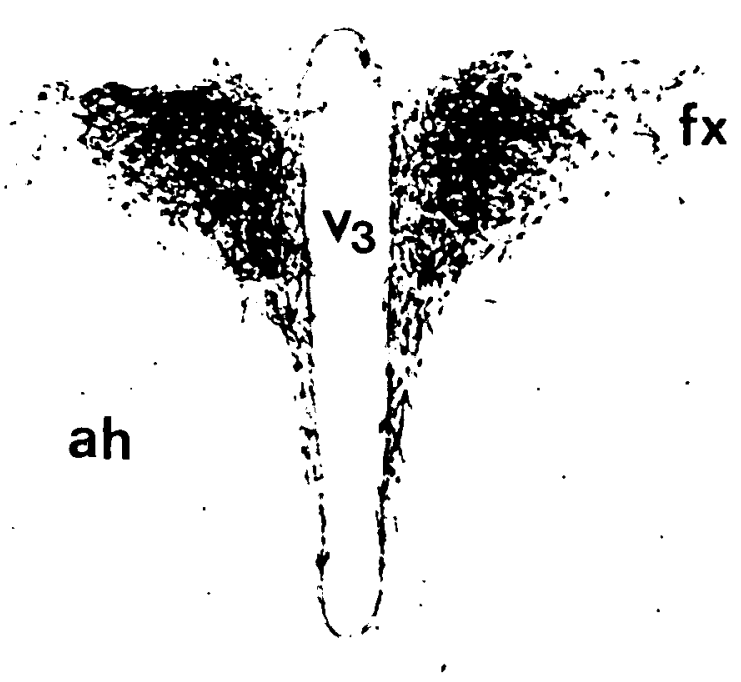

Figure $13-C R F$-immunoreactive neurons in the paraventricular nucleus. Perikarya fill both parvo- and magnocellular portions of the nucleus. Compare this distribution with opiocortin fibers in Fig. 3. X35.

OPIOCORTIN BIOSYNTHESIS

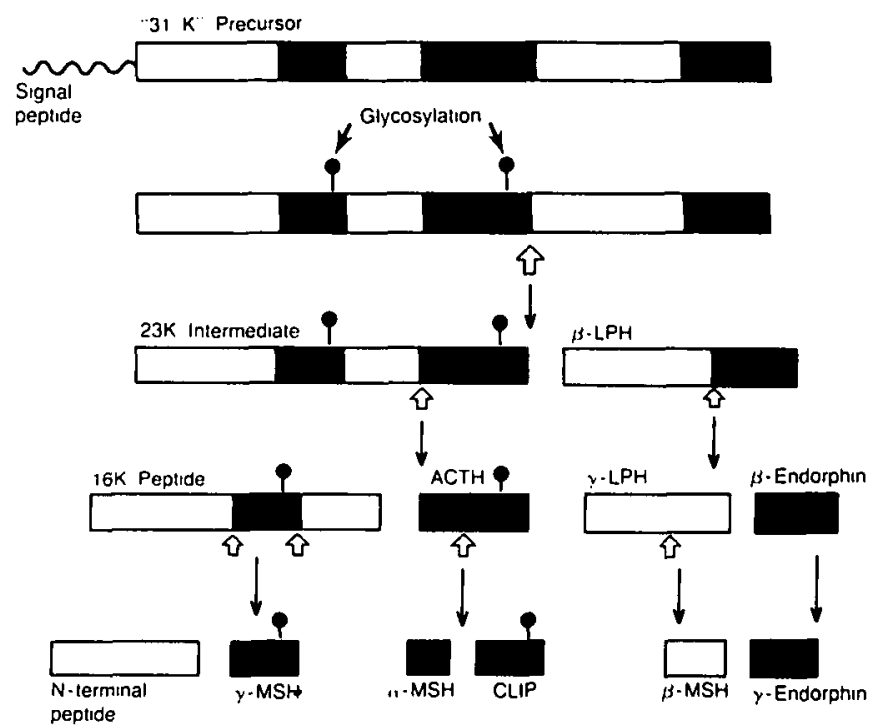

Figure 15 - Diagram of the biosynthetic pathways for formation of the opiocortin peptides. 


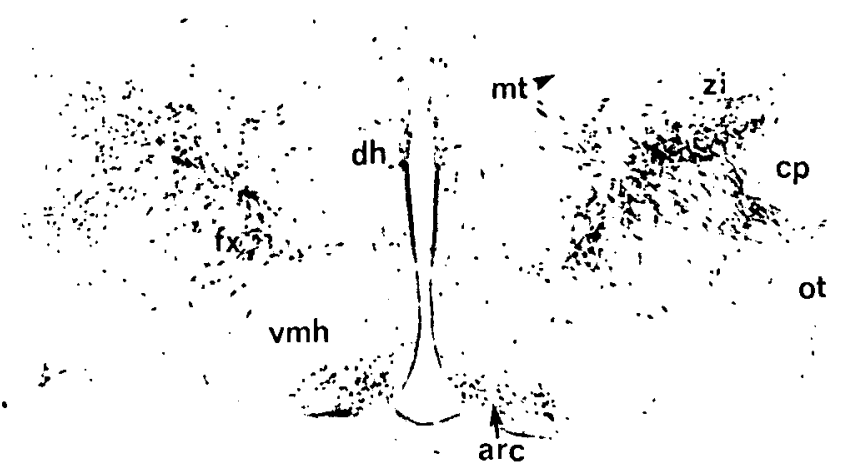

Figure 16 - Frontal section of rat brain immunostained with anti-alpha MSH. Perikarya of the arcuate opiocortin system are demonstrated in the arcuate nucleus (arc). Alpha MSH-specific neurons occupy a vast area of the lateral hypothalamus; at this level, perikarya of this system cap the fornix $(f x)$, the cerebral penduncle (cp), and extend far into the zona interna (zi). X20.

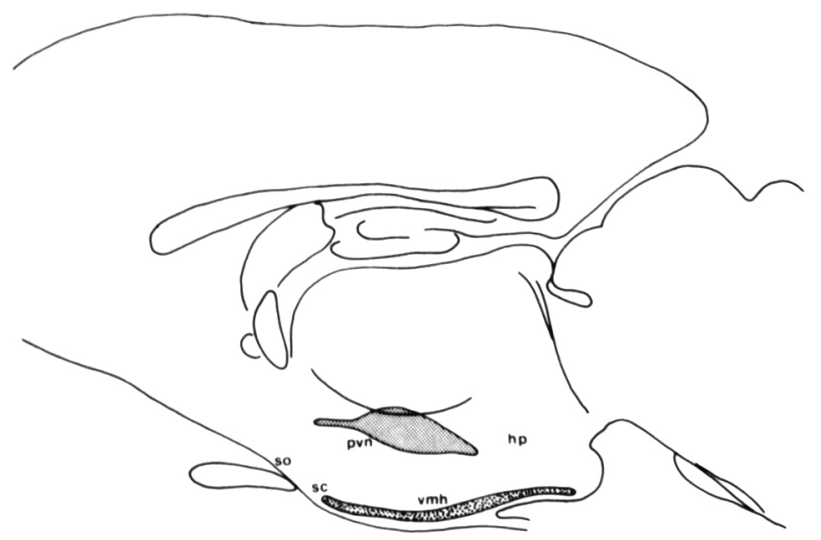

munoassay as well as bioassay data, the 3 active peptides -ACTH, $\beta$-endorphin, and $\alpha \mathrm{MSH}$ - appear to be present, at least in the perikarya of arcuate opiocortin neurons. The recently discovered brainstem medullary opiocortin neurons have been described anatomically on the basis of their immunoreactivity to anti-ACTH and anti-16K peptide; details of their peptide profiles awaits further study. Immunochemical studies based upon antibodies generated against the opiocortin peptides must be viewed with caution in these considerations because of possible cross-reactivity with their parent sequences, their respective intermediate products, and their common $31 \mathrm{~K}$ precursor. Numerous challenging and physiologically important questions about the peptides of the central opiocortin systems remain to be answered. The magnitude of these systems in the brain and their diverse distribution imply unmistakably a role in a wide variety of functions in pain perception, motor function, and hypothalamic, behavioral and autonomic neuroendocrine circuits. While $\beta$-endorphin is established without question as a major opiocortin neurotransmitter, it remains to be seen whether it is the only one used. Several anatomical as well as physiological studies have suggested a functional sub-organization of the pool of neurons of the arcuate opiocortin system, implying that there may be discriminative activation of subunits of neurons which selectively innervate hypothalamic nuclei, amygdala, or discrete brainstem regions. If such data suggests varied afferent synaptic organization, than the possibility exists that these circuits may employ different opiocortin peptides as their major neuroeffectors.

The alpha MSH-specific or $\alpha-2$ system represents the third and perhaps most intriguing of the opiocortin neuronal subgroups. First recognized in the rat brain by Watson and Akil in 1979, this component consists of a community of neurons whose perikarya are located in the lateral and dorsal areas of the hypothalamus, totally separate and without overlap with the arcuate opiocortin perikarya (Figs. 16, 17), and with unique immunochemical characteristics. Our studies (Knigge and Melrose, unpublished) indicate that the cell bodies of this system extend from the level of the optic chiasm to the junction of the posterior hypothalamus and mammillary body. Anteriorly, the cells are arranged in a thin horizontal band, extending from the midline laterally into the zona incerta. The field of cell bodies enlarges markedly at the level of the paraventricular nucleus, virtually filling the upper half of the hypothalamus. Further posterior, the location of the perikarya is shifted laterally; they encircle the fornix, fill the lateral hypothalamus and cap the cerebral peduncle. Projections from this system are directed to the hippocampus and cerebral cortex. A fairly dense plexus of fibers uniformly fills all layers of the frontal, parietal, temporal, and occipital cortex and the olfactory bulb. Both dorsal and ventral hippocampus, subiculum and dentate gyrus are innervated. These neurons are immunoreactive only to antisera generated against synthetic $\alpha \mathrm{MSH}$; in neither normal nor colchicine-treated animals are we able to stain this system with antisera against $\mathrm{ACTH}, \beta$-endorphin or the $16 \mathrm{~K}$ intermediate peptide. The exact identity of the $\alpha \mathrm{MSH}$-like peptide in this system and its biosynthetic pathway is at present unknown. Functionally, this $\alpha \mathrm{MSH}$ system may represent an exceptionally interesting pathway for the discharge of hypothalamic influences upon the hoppocampus and cerebral cortex. The uniformity of its distribution to these areas suggests a generalized rather than specific effect. Alpha MSH and other neuropeptides have been implicated in a variety of brain processes concerned with memory and behaviour; whether this hypothalamocortical $\alpha \mathrm{MSH}$ system represents the morphological substrate for such activity remains to be determined.

\section{ACKNOWLEDGEMENTS}

The authors beg the indulgence of their many colleagues and collaborators who have contributed more than they to the anatomy and physiology of the opioid systems of the brain. Lack of space, not lack of respect, dictated the omission of this large volume of literature.

\section{REFERENCES}

Joseph, S.A. (1980). Immunoreactive adrenocorticotropin in rat brain: A neuroanatomical study using antiserum generated against synthetic ACTH ${ }^{1-39}$. Am. J. Anat. 158:533-548, 1980.

Joseph, S.A. and Knigge, K.M. (1983). Corticotropin releasing factor (CRF): Immunocytochemical localization in rat brain. Neuroscience Letters 35:135-141

Joseph, S.A., Pilcher, W.H. and Bennett-Clarke, C. (1983). Immunocytochemical localization of ACTH-ir perikarya in nucleus tractus solitarius in the medulla: Evidence for a second opiocortin neuronal system. Neuroscience Letters 38:221-225.

Knigge, K.M., Joseph, S.A. and Nocton, J. (1981). Topography of the ACTH-immunoreactive neurons in the basal hypothalamus of the rat brain. Brain Res. 216:333-341.

Knigge, K.M. and Joseph, S.A. (1981). Relationship of the central ACTH-immunoreactive opiocortin system to median eminence and the pituitary gland of the rat. Cell Tiss. Res. 215:333-340. 
Knigge, K.M. and Joseph, S.A. (1982). Relationship of the central ACTH-immunoreactive opiocortin system to the supraoptic and paraventricular nuclei of the hypothalamus of the rat. Brain Res. 239:655-658.

Michael, G.J. and Joseph, S.A. (1983). Analysis of efferent projections from the arcuate opiocortin system in rat brain. Soc. for Neuroscience Abstract, Boston, Mass.

Pilcher, W.H. and Joseph, S.A. (1983). Co-localization of CRF-ir perikarya and $\mathrm{ACTH}$-ir fibers in rat brain. Brain Res., in press.
Romagnano, M.A. and Joseph, S.A. (1983). Immunocytochemical localization of $\mathrm{ACTH}{ }^{1-39}$ in the brainstem of the rat. Brain Res. 276:1-16.

Sawchenko, P.E., Swanson, L.W. and Joseph, S.A. (1982). The distribution and cells of origin of ACTH (1-39)-stained varicosites in the paraventricular and supraoptic nuclei. Brain Res. 232:365-374.

Scott, P.M. and Knigge, K.M.. (1981). Immunocytochemistry of luteinizing hormone-releasing hormone, vasopressin and corticotropin following deafferentation of the basal hypothalamus of the male rat brain. Cell Tiss. Res. 219:393-402. 